\title{
FROM THE ELABORATION PROCESS OF POINT CLOUD TO INFORMATION SYSTEMS BOTH FOR PLANNING AND DESIGN MANAGEMENT OF CULTURAL HERITAGE
}

\author{
P. A. Ruffino ${ }^{1}$, M. M. Bocconcino ${ }^{1, *}$, M. Del Giudice ${ }^{1}$, A. Osello ${ }^{1}$ \\ ${ }^{1}$ Dept. of Structural, Geotechnical and Building Engineering - Politecnico di Torino, Turin, Italy (pablo.ruffino, \\ maurizio.bocconcino, matteo.delgiudice, anna.osello)@polito.it
}

KEY WORDS: Urban LOD, Scan-to-GIS-to-H-BIM, HGIS, HDIM.

\begin{abstract}
:
Nowadays we are able to produce geometric models of historical building at different scale of detail using photos and measurements. More and more we are facing with lack of preservation actions and maintenance activities, bad foreseen policies, unexpected natural events, that are forcing professionals and researchers to operate without usual data. In these cases, we need consistent repository to collect and distribute data to produce information. Furthermore, we need to "give intelligence" to these repositories in order to query them with respect geometrical instances, topological issues, historical features.

We dispose of tons of xyz points: how can we pass from the point cloud to a building information model, then to a geographic information system, not necessarily in this order? A simple Scan-to-BIM-to-GIS and Scan-to-GIS-to-BIM process were tested in order to consequently evaluate, with purposes of preservation and of enhancing of resilience, some practices that could became the best, also in terms of time and cost saving.

The work we propose is a part of an ongoing research focused on the application of H-BIM approach for the management of historical building heritage, focused on a district management (H-DIM, at an urban level). In particular, with regard to the resilience theme, both the acquisition phase and the archive research process are of great importance for protecting our undefended building heritage.

Regarding the case study of the paper, UNESCO sites represent important areas for collective interests of humanity. This contribution proposes a possible solution applying a digital cultural heritage to the historical part of the Municipality of Serralunga d'Alba belonging to the UNESCO site called Vineyard Landscape of Langhe-Roero and Monferrato.
\end{abstract}

\section{INTRODUCTION}

In recent years, LIDAR and image-based (photogrammetry) systems are widely using by professionals in the field of geomatics. These geospatial detection systems allow to obtain dense three-dimensional measurements in a very short time (Zhang et al., 2018).

The point clouds obtained allow us to describe the environments surveyed with accurate precision that are very useful in the modelling of built heritage. In this context, the segmentation and semantic interpretation of 3D point cloud is one plays an essential role during 3D modelling phase (Hernàndez and Marcotegui, 2009). Depending on the size of the survey, this post-processing operation can consume a quantity of time that reduces the time advantage given by the geomatics instruments available today. For this reason, the scientific community of the computer science sector dedicated to the digitization of the built environment continues to address the automatic classification issues of the point cloud (Hron and Halounová, 2014; Thompson and Boehm, 2015).

As far as the surveys of urban environments are concerned, many softwares make easy to classify point clouds through semiautomatic classification tools that make possible to distinguish points which belong to vegetation, ground and buildings. In some research projects, algorithms have been developed to identify also the points belonging to the cars, pedestrians and elements of urban furniture and even power-lines and pylons (Guo et al., 2015).

In this sense, it is important to mention another theme that is very much discussed in this field: the automatic generation of 3D models from the point cloud (Xu et al., 2004; Pang et al., 2015; Poullis, 2013). Most of the solutions use tools and algorithms that refer to the RANSAC method (RANdom SAmple Consensus) developed by Fischler and Bolles in 1981.

This system allows you to find the primitive geometry from a point cloud. Without entering into the merit of the achievable accuracy, basically the system is very useful but has important limits to consider. In the field of urban modelling, this system allows you to create a model for each building detected only if the buildings are separated from each other. In other words, the automatic 3D modelling of a historical village made up of $n$ buildings joined together, generates a single $3 \mathrm{D}$ model. In addition, all models generated are simple 3D solids and can only be used for graphic and visualisation purposes. Consequently, these methodologies are not suitable for the issues of management and conservation of the built heritage.

Another approach suggested by the literature is the Digital Cultural Heritage developed through HBIM (Historic Building Information Modelling) methodology. It aims to get the digitalisation of existing architectural heritage through BIM approach starting from geospatial data (Dore and Murphy, 2012). In this case, the platforms dedicated to parametric object-oriented modelling are the most suitable tools for generating parametric models (Tommasi et al., 2016). In fact, this methodology represents the system that allows the convergence of geospatial data, 3D modeling and alphanumeric information in a single work environment.

In the following chapter it will be considered an approach that

\footnotetext{
* Corresponding author
} 
lead form a list of xyz coordinates to a classification of the points consequently to their spatial join with areal shapes. Then, an information model is developed, constantly linked with a geographic information system and the other way around.

\section{POINT CLOUD AS A FIRST STEP OF AN INFORMATION SYSTEM}

\subsection{Integration and interaction of geospatial data}

Nowadays we are able to produce geometric models of historical building at different scale of detail using photos and measurements. More and more we are facing with lack of preservation actions and maintenance activities, bad foreseen policies, unexpected natural events, that are forcing professionals and researchers to operate without usual data.

In these cases, we need consistent repository to collect and distribute data to produce information. Furthermore, we need to "give intelligence" to these repositories in order to query them with respect geometrical instances, topological issues, historical features.

The contribution proposes methodological and operational aspects related to the path that from the point clouds identified by position and colour and other attributes leads to the information management of data in dedicated processing environments.

Automation aspects will be highlighted in relation to the treatment of the regions of the point cloud and with respect to their semantic classification starting from descriptions "at level zero", up to more in-depth level descriptions and management within complex information systems.

Efficiency issues in term of integration and interoperability between different technologies implies to combine contents associated with different scale of representation, generalize them and establish processes without any solution of continuity from informative system at territorial level, passing through the urban one till the building information model (Figure 1).

This last aspect, (archives and databases connected with geometrical issues), leed our digital model to a new dimension, the informative one (where spatial, temporal, historical and building parameters work together), that should always characterize speculative actions towards the constitution of a wealth of knowledge: we need to work on efficiency of the process to reach effectiveness of methodologies of survey. And we need to involve people (institutions, professionals, users and common people) to feed knowledge through dedicated and specialized (ad hoc) social network: query repository of data to produce new information.

\subsection{Data elaboration and knowledge processes}

The work we propose is a part of an ongoing research focused on the application of H-BIM approach for the management of historical building heritage. In particular, with regard to the resilience theme, both the acquisition phase and the archive research process are of great importance for protecting our undefended building heritage.

We dispose of tons of xyz points: how can we pass from the point cloud to a building information model, then to a geographic information system, not necessarily in this order? Scan-to-BIMto-GIS and Scan-to-GIS-to-BIM processes will be compared in order to consequently evaluate, to purposes of preservation and of enhancing of resilience, some practices that could became the best, also in terms of time and cost saving (Boschè et al., 2015). In order to evaluate possible answers and them efficacy, starting from the state of the art synthetized in the previous chapter, some aspects of automatization in this process will be considered, using for instance spatial query that are prerogative of a GIS, and using topological relationship between building components set in a BIM environment, with the aim of combining in a unique spatial database data and information that sustain different preservation purpose at different scale of intervention (Malinverni and Tassetti, 2013). Architectural and building information modelling systems do not allow "spontaneous native management", spatial themes and interrogations. That is, they must be mediated by appropriate computer equipment that extracts the information and graphically represents it according to certain representation and communication codes.

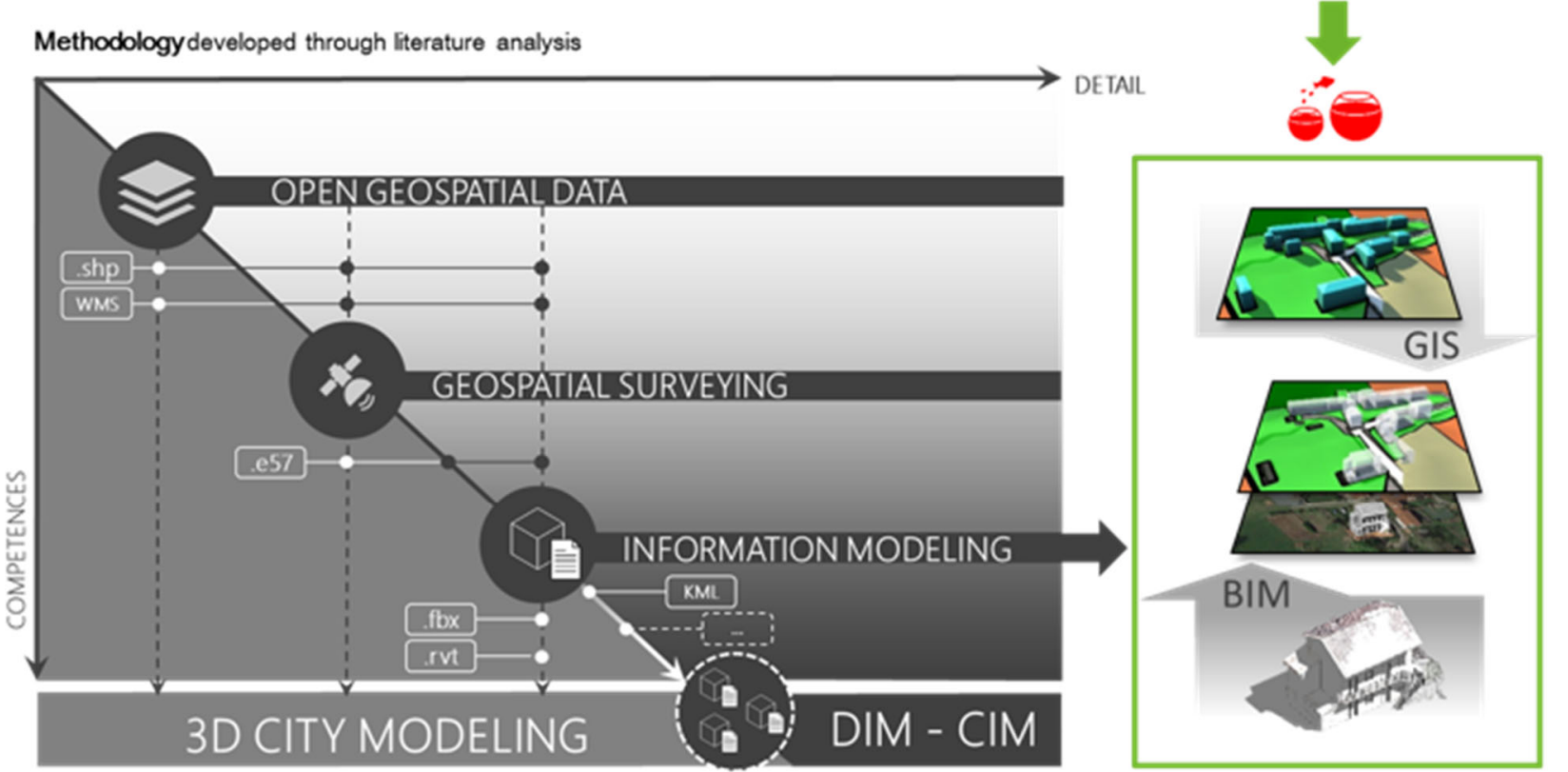

Figure 1. Built heritage digitalization process 
The methodological frameworks that must be prepared also require specific skills, human resources trained in programming and calculation within the operating environments.

Geographic information systems, on the other hand, are more dedicated to the spatial interrogation and overlapping of different thematic levels that can also be properly weighed.

The GIS open the front of the BIM to the urban representation of specialized type, preserving all the building components useful for the management towards larger scales of data management.

The methodology of data processing and representation proposed aims to provide management approaches open to the largest number of skills involved in the conservation and protection of environmental and cultural heritage (Osello and Rinaudo, 2016). An open process in fact allows to incorporate every point of view of the professional system, not excluding some of them because of technological barriers not directly accessible.

In this sense, web-like sharing environments can bring added value and seamlessly within the processing containers of the parametric digital model, thus maintaining IT quality and broad participation to different stakeholders (Kang et al., 2016).

More specifically, using point cloud as input data to get $3 \mathrm{D}$ models, we have deal with restitution problems of historic buildings: crooked walls, overlapping roofs which belong to different buildings, irregular geometries, and so on.

In addition, we have learned how to manage information models, simplifying the interpretation of information according to data needed and fixed from the start.

\subsection{Case study: the UNESCO site of Serralunga d'Alba}

With regard to the case study considered for this paper, The United Nations Education, Scientific and Cultural Organization (UNESCO) sites represent important areas for collective interests of humanity. They are conveniently protected by international community to conserve and preserve their features for next generations. In this contest, a fundamental aspect is the promoting of these places and landmarks through innovative communication and visualization technologies (Spallone et al., 2016).

The UNESCO defines cultural heritage as "the legacy of physical artifacts and intangible attributes of a group or society that are inherited from past generations, maintained in the present and bestowed for the benefit of future generations".

Protecting these places and landmarks means to preserve both tangible and intangible goods: historic places, monuments, artifacts together with the culture and customs to which they belong (Adler and Urice, 2011). UNESCO also identifies a list of factors affecting the value of world heritage properties: observing them it is easy to understand that human activity represents the main factor interacting with cultural heritage sites. So, the relations of all actors who interact with cultural heritage have to be governed carefully.

In this contest, public bodies and private foundations devoted to promoting of world cultural heritage have to operate through specific activities of preservation, conservation, management and dissemination (Sullivan, 2016).

This site represents an extraordinary landscape which is the result of an awesome relationship between human activities and nature. It is characterized by vineyards together with historic buildings and villages developed according to 'wine tradition'.

The site is composed of 6 'core zones' which include 72 towns in more than 10.000 hectares. These areas are protected by a 'buffer zone' of about 76.000 hectares with 72 towns. Given the vastness of the territory, only the historic village of Serralunga d'Alba has been used for the project development.

This village is composed of an historical part developed around a castle of 14 th century and it is encircled by a vineyard landscape of great environmental value.

As mentioned before, project is developing using HBIM methodology, toward a district management (HDIM).

It aims to get the digitalisation of existing architectural heritage through BIM approach from scan or photogrammetric survey data.

In order to follow this workflow, the first step has been the survey phase of external parts of buildings studied.

Given the morphology of the village, the survey was combined using both photogrammetry survey taking pictures by a commercial UAV (Unmanned Aerial Vehicle) and an innovative laser scanner called "Leica Pegasus Backpack".

Drone was used to survey whole village: this allowed to get a point cloud with a good accuracy concerning the context, the castle and roofs (Chiabrando et al., 2017).

But this approach did not allow to get geometric data into narrow streets. For this reason, it was tested a wearable mobile scanning solution: this innovative geospatial instrument has expedited survey operations keeping the output data with highperformance.

A GNSS (Global Navigation Satellite System) survey carried out too in order to use the same global reference system and to match both point clouds generated by photogrammetry and scan technologies.

This operation was made into Autodesk ReCap software getting the main input geometric data to design BIM models of the Municipality of Serralunga d'Alba (Figure 2).

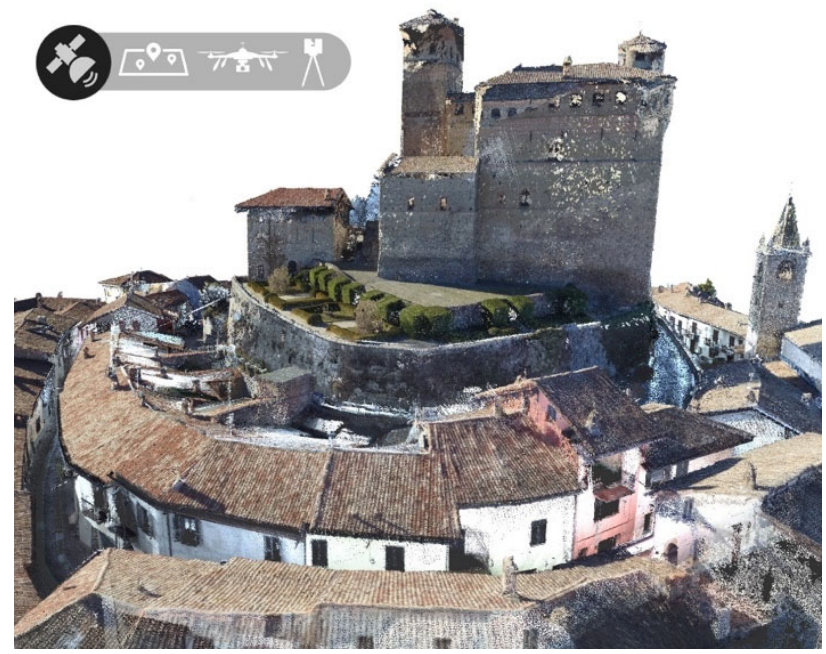

Figure 2. Point cloud obtained by geomatic survey

Regarding the castle, specific information model has been created to help the preservation and management goals of the landmark (Figure 3).

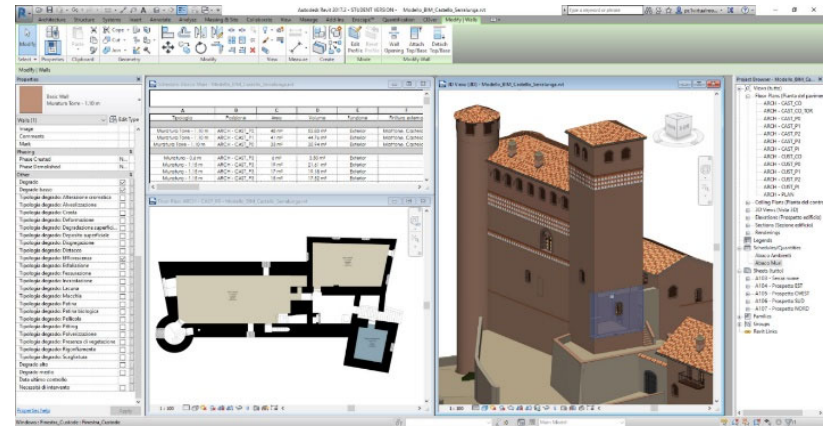

Figure 3. HBIM of the Castle 
More specifically, BIM components contain data about the state of material conservation and what kind of intervention has to be done. Moreover, information model can be useful for manage spaces according to Facility Management (FM) method (Aicardi et al., 2018).

In order to create an information model of Serralunga, next step was to get a point cloud for each building through the classification phase. Thus, it was created a federated model composed of a coordination model where there are linked BIM models of individual buildings (Figure 4).

A complex information model at territorial level has been developed. Three-dimensional model was used as geometric data input for developing of immersive visualisation systems (Ruffino and Del Giudice, 2018).

Another previous paper edited by one of the authors has proposed possible solutions applying a digital cultural heritage to the historical part of the Municipality of Serralunga d'Alba belonging to the UNESCO site called Vineyard Landscape of Langhe-Roero and Monferrato (Ruffino, 2018).

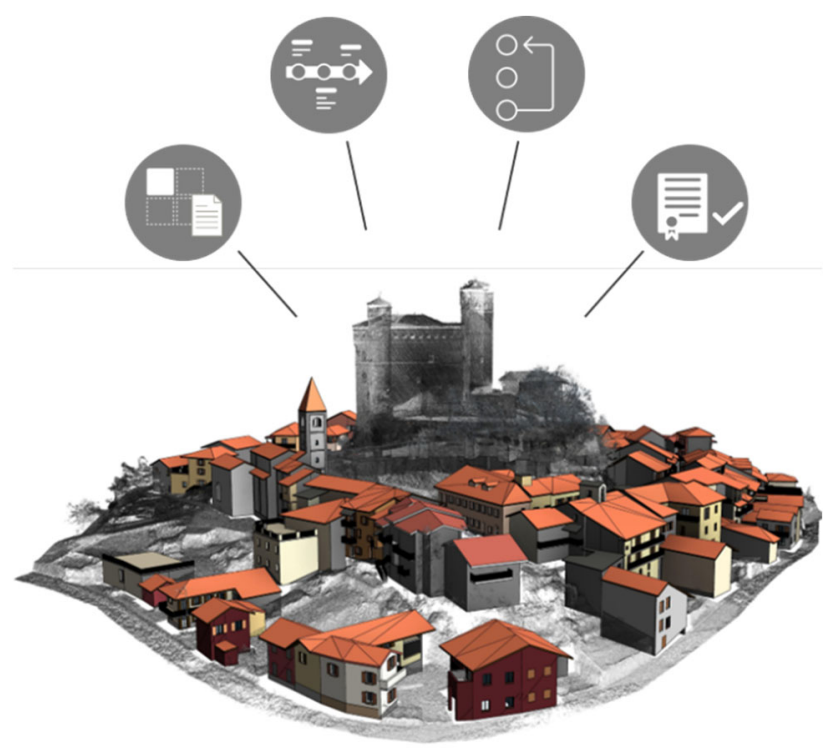

Figure 4. HDIM of the historic Municipality of Serralunga d'Alba

\subsection{Building level point of view}

Representation and its techniques play a fundamental role in order to transmit knowledge. We specifically join the geometrical model to a clever archive of data (families of structured and well known elements, intended as the minimal logical part of the whole building). After having put into an interoperability mode a relational database with repository of "intelligent objects", we create a procedural path that allows users (researchers and professionals) to:

- interactively select an element of the repository (thanks to a query builder);

- $\quad$ import the BIM family into the BIM project;

- $\quad$ associate the elements (Building Object Model BOM) to the digital geometrical model;

- $\quad$ link other parameters to elements (alphanumerical and iconographic documentation; conjectures, other sources);

- $\quad$ perform a semantic validation of the H-BIM.

We have considered also the possibility to distribute database and query builder starting from a web app using computer technology ordinarily used for social internet site platforms (not discussed in the present paper). This digital model was used as a reference to develop an H-DIM as shown in the next paragraph; it demonstrates the feasibility of a whole H-BIM approach for complex urban context, starting from point clouds. In order to improve the framework for 3D modeling, the experience has shown the challenges of using integrated procedures to organize documentation when the information has been normalized.

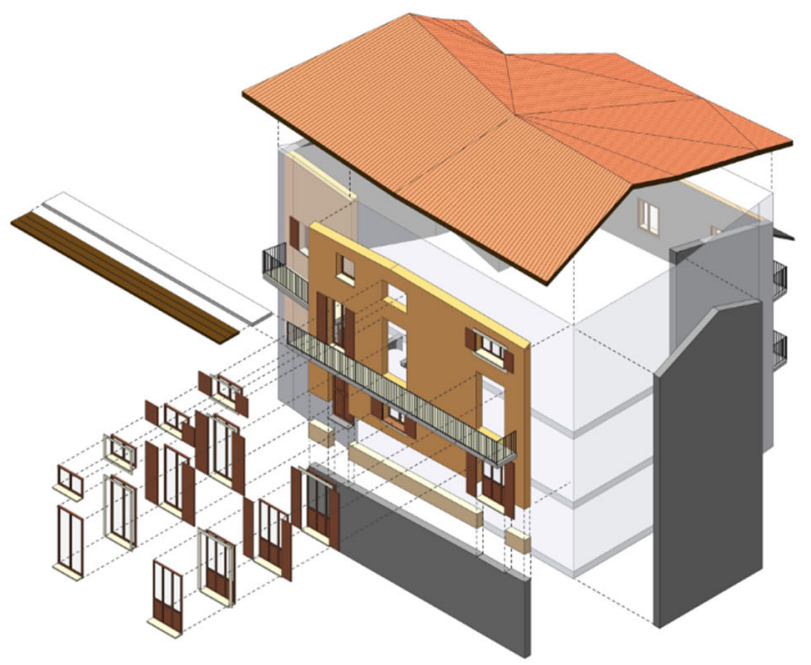

\section{LOD 3}

COMPONENTS DATA

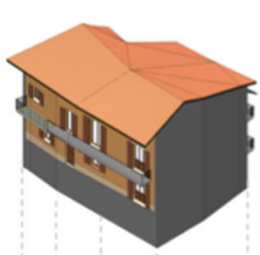

Family

Type

Building code

Orientation

Dimensions

Phase

Materials

\section{LOD 1}

BUILDING DATA

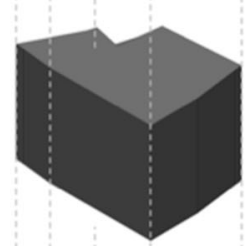

Building code

Building typology

Environment interest

Action state

Action expected

\section{LOD 0}

CARTOGRAFY DATA

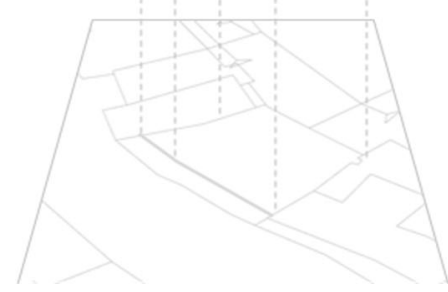

Figure 5. From Level Of Development (LOD) applied to building level to LOD applied to urban level

The main objective is creating an interoperable and queryable three-dimensional models of the built heritage where the scholars can access and conduce comparison between similar case studies, directly or by analogy, and then associate the results of the document search with specific parts of the model (Figure 5). 


\subsection{Urban level point of view}

A large part of the resources of the processes that lead to the semantization of the geometrical points (measured as the position of material elements in a real context), up to the management of those data with specific purposes (of protection, active conservation and urban planning, with aspects of the specification of policies for the protection and management of the historical building heritage), is used for the manual identification of the volumes and surfaces that must be traced back to a particular building or to another in order to allow its information modelling (Lo Turco and Bocconcino, 2017).

The phase presented here aims to make the process of associating points to a given building unit more efficient, accepting margins of gross error that should not prejudice the aspects of subsequent modelling, relying on the competence or ability to discriminate of those who produce it (Tobias, 2015).

Not only that. In addition to a mere aspect of efficiency in terms of processing times, this processing phase introduces a not insignificant element for the purposes of data management; in fact, it allows to uniquely associate the building to each point detected. This association can be very useful for all those operations of alphanumeric interrogation and subsequent reworking.

The idea it supports is precisely that of uniquely naming the geometric points detected through the identification of the building unit or, in any case, of the geometric entity of reference for that point, of its afference.

The sequence of elementary operations that has been defined derives from the consideration of having to make directly experimentable, in a simple way and accessible to specialized users, the continuum process that leads from the point cloud, intersecting the geometric-parametric data with specific cartographic and planimetric themes, to the information model according to progressive refinement LODs, considered at the level of urban scale (Vacca et al., 2018a, 2018b).

The technologies involved can be traced back to four types of applications:

A. those dedicated to the treatment of point clouds, in particular prepared for the export in tabular format of rows of points defined by their position in space and by related attributes associated by the instrument that detected them (colorimetric or related to other parameters associated with the property of the surfaces and materials concerned);

B. those specific to the processing of alphanumeric data (spreadsheets and multirelational databases);

C. those relating to geographic information systems and to spatial and alphanumeric interrogation;

D. those specialized in building information modeling processes.

The basic data for the treatment are as follows:

- $\quad$ point cloud detected (it is not interesting here to specify sampling methods and techniques);

- planimetric cartographic themes in areal format (technical cartography of urban level with identification of building cells).

- The steps can be listed as follows:

- processing of the point cloud in order to extract in tabular form the parameters of position and characterization of each point ${ }^{1}$;

- $\quad$ import in GIS environment of the list of "points", correctly georeferenced;

\footnotetext{
${ }^{1}$ Given the large number of points involved, thinning techniques must be used appropriately; with regard to the spatial interrogation that must follow, carried out on the horizontal
}

import of cartographic themes into GIS environment (e.g. "buildings" perimeter with related attributes table);

- $\quad$ spatial interrogation that searches for points that fall within a significant perimeter of the perimeter of individual buildings;

- transfer of the identification parameter from the "buildings" table to the "points" table;

- $\quad$ export building by building of the relevant point cloud;

- reassociation through join operations of the values relative to the altimetric altitudes measured;

- import into the BIM environment for information modelling according to progressive refinement LODs (Deng et al., 2016).

This procedure uses only functions native to the technologies, without computer programming aids (Ma and Ren, 2017); moreover, it suffers a gross error of association for all those points that belong to roofs or projecting elements that do not adapt to the geometry of the ground system of the building and therefore generate inaccuracies in connection with the corresponding area geometry.

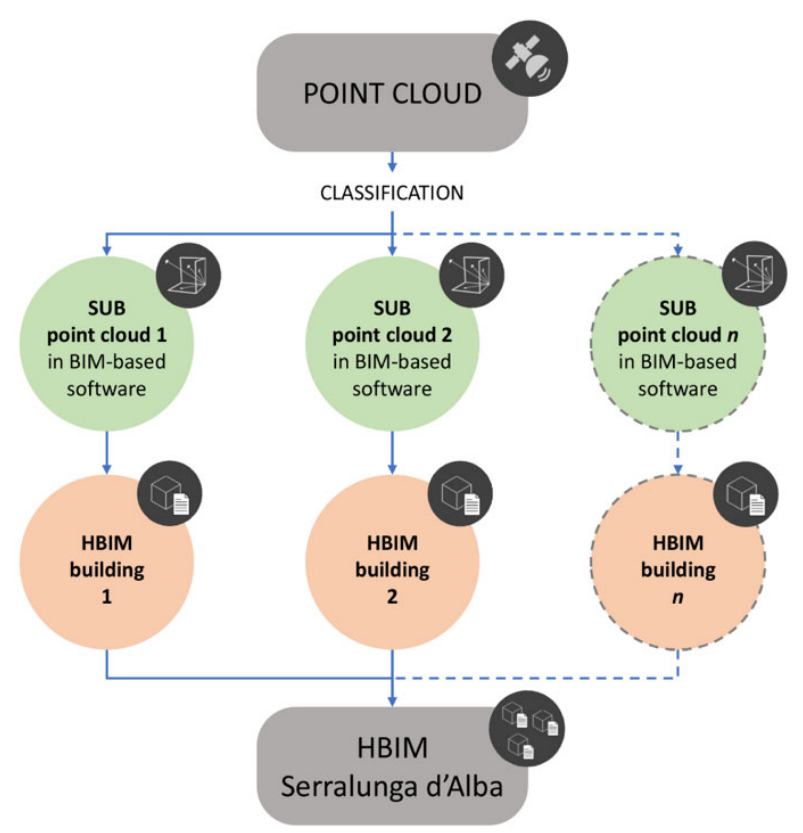

Figure 6. Federated model of the HBIM applied to urban level

A possible web platform set for diffusion must consider several aspect to take into account: the creation of complex databases of the building must follow the guidelines set out by the Central Institute for the Catalog and Documentation (ICCD) of the Ministry of Cultural Heritage and Tourism (Santoro et al. 2016). The platform must be easy-friendly to facilitate participation of an enlarged public (institutions, scholars, professionals, citizenship); raising public awareness of the need to supply historical data archives through accessible and transparent tools; the ability to interrogate databases differently according to different search filters that can be combined (Donato et al., 2017). From the above-described requirements framework, the following operational tools (in a prototype version) have descended on the verification of the methodological approach we have outlined. In particular, this platform, which has been

xy plane, the reference $z$ value equal to the value 0 will be set fictitiously, re-associating the identification values at a later date, restoring the real values of altitude. 
decided to operate on distributed computers and, on the other hand, on the web, includes two spaces of interaction: one dedicated to the inputs of the database, open to a widespread, appropriately accredited public; The other turned to certain profiles that work on the digital reconstruction of the built.

The open virtual public square provides archiving tools of various types of materials, metadocumented according to what is foreseen in the catalog of cultural goods. It allows the correct sorting of digital materials, functional to the consistent questioning of the sets (Previtali and Latre, 2018).

In more detail, those who work on the building information system provide appropriate parameters for the objects of the model library (general, geographic, and typographical, typological, geometric, topological attributes); the input of these parameters allows their conjunction with "and" and "or" constructs in a query string that is made up of a specific hypertext parameter called "search".

The search parameter could be compiled within a relational database that has the specific purpose of integrating specific data operations, which are not possible within the BIM processing environment. Specifically, the system interacts with model library objects (BOMs), or with its specific formalizations (areas, volumes, or masses) (Figure 7).
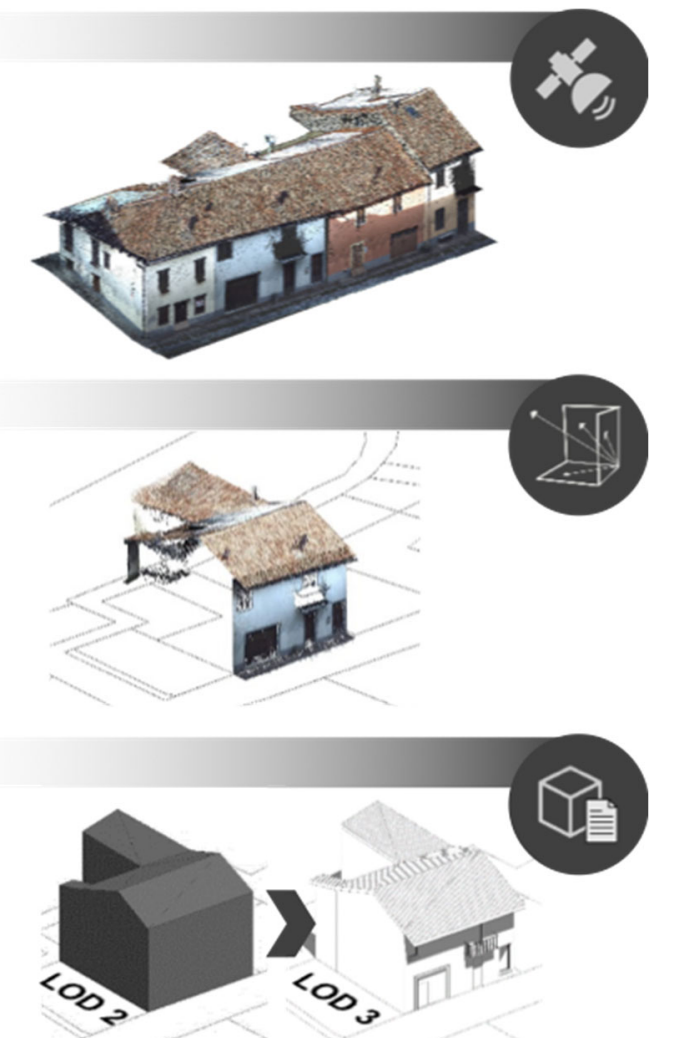

Figure 7. Information modelling workflow

The widespread application of the survey method and graphical representation of resilience aspects of urban context requires the system to be set up of a series of investigative and restitution elements.

On the one hand, the progressive refinement of the parameters to be detected - and the way in which these could be synthetically collected and represented with "a single glance" - was supported by processing tools that today we consider to be "artisanal", manually set up and edited until the correct proportions are established between symbols, annotations and graphic signs.

On the other, the need to make consistent the number of case studies to be submitted to verification and standardization of the use of graphic rules, requires the preparation of specific protocols, this time of computer type, which allow to delegate to the computer the aspects related to the graphic synthesis and analytical weighting of the results of the relief survey (Figure 8). The parametric information model "records, archives, preserves" and "represents, simulates, foreshadows" at the same time. It does so at the same time as we operate, it reflects changes and variations in real time. For this reason, a substantial part of the time dedicated to the preparation of the model is entrusted to the study and preparation of the graphic codes and to the sensitivity of the representation.
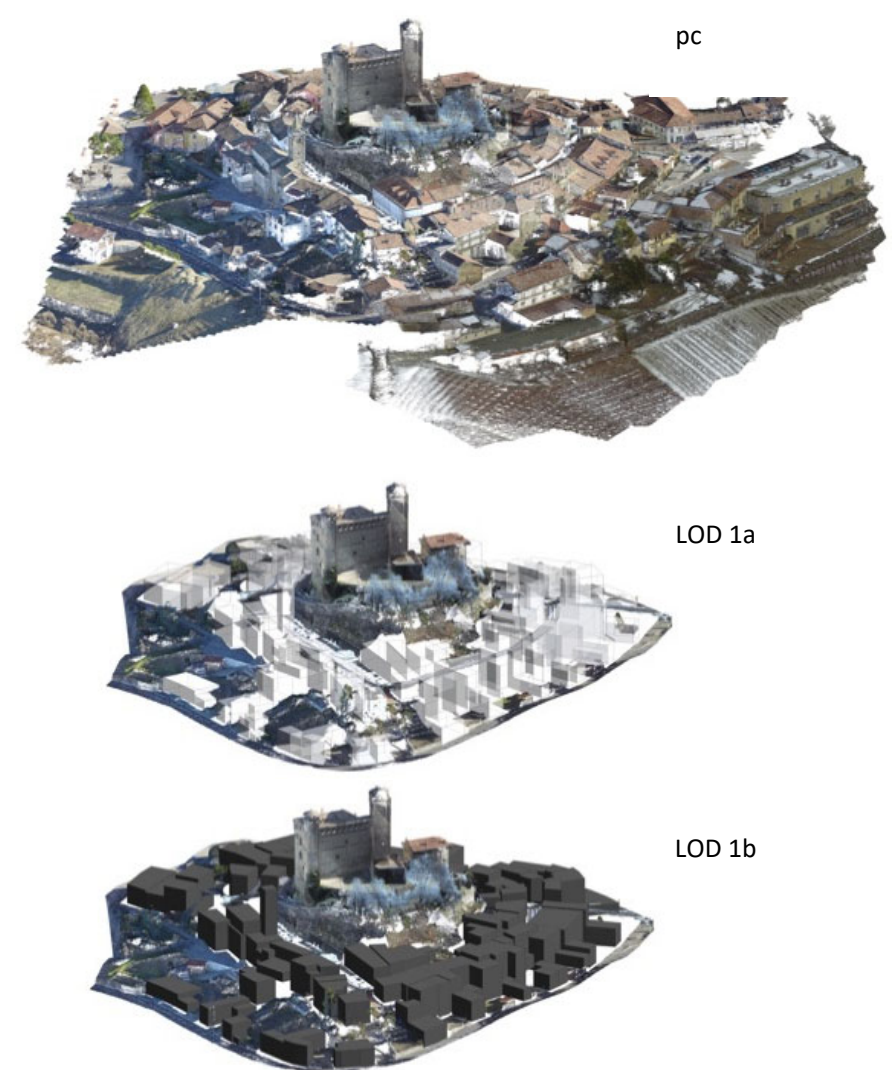

LOD $1 b$

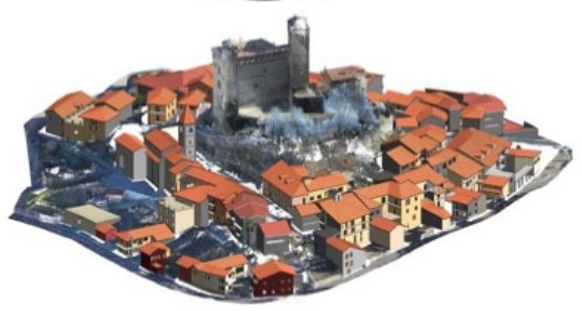

LOD 3

Figure 8. LOD refinement and knowledge progression

\section{CONCLUSIONS}

The use of information solutions, with both geometric and parametric values, is of interest today to all those who work in the territory in areas such as planning and programming, civil protection and general safety, environmental management, location and protection of assets, tax administration, management of routes and logistics in general, the management of technological networks for companies, the location of companies, the conduction of project work, research in the field of archaeology. Many of these initiatives offer addenda in terms 
of services that are essentially web-oriented, i.e. they use webmapping ${ }^{2}$ and web-GIS ${ }^{3}$ solutions accessible from fixed and mobile workstations.

The meeting of people around a common theme within virtual spaces that allow interaction and comparison, can produce sharing, knowledge, heterogeneity of views in the unity of intent (at least in one of the many desirable societies), clashes and frictions (where the aims differ or are not clearly expressed), but it is also useful to the growth of knowledge. After all, therefore, it is precisely the sharing, dissemination and reproduction of virtuous practices that generates progress and change (Figure 9).

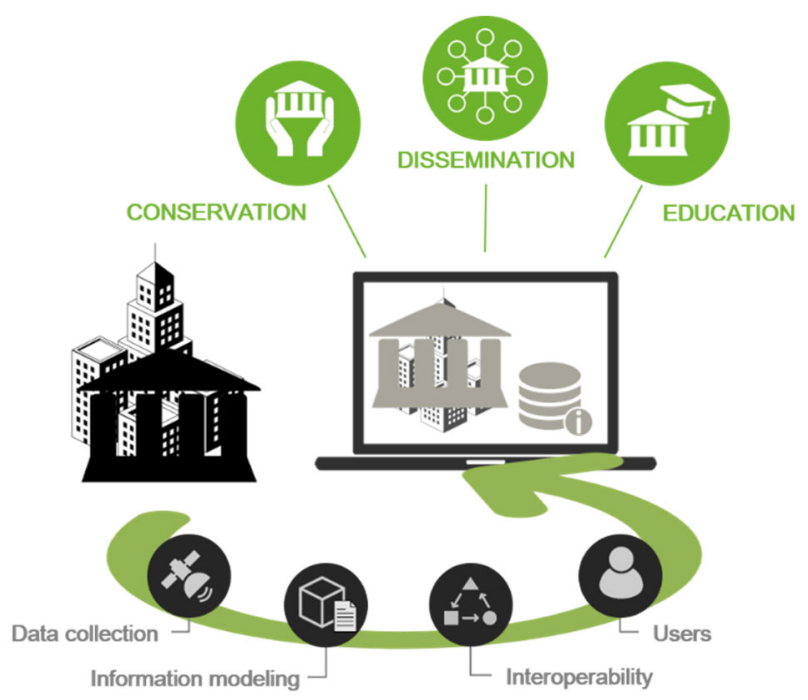

Figure 9. Data flow, users and purposes

In the early stages of innovation, it is always technology that attracts attention. Then, however, the speed of change leads to a loss of sight of what could be the subsequent positive effects of technological progress itself. The relationship between the use of technology and the culture of data is actually of vital importance because technology requires organization and the latter cannot exist without a culture of organization of data and the tools to represent them.

The research conducted until now, demonstrate the potential of H-BIM applied for driving the process of reconstruction after seismic event due a loss of information (Figure 10).

Although the procedure show high potential in general, from another perspective show limits especially connect with the difficulties of populating a virtual library of case studies and elements (BOMs for historical building heritage).

Further work will be conducted in presenting officially the platform and start with the phase of "sensitization" and gathering of information for developing the third version of the model.

The final objective will be to achieve a complete urban model including all the architectural technical notices necessary to guide the preservation and conservation.

Another expected goal is the iterative work of defining the graphic codes, application to different scales in-depth analysis,

2 Web-Mapping, or better web-map-service, is a specific technique defined by the OpenGIS Consortium (an international non-profit organization that defines specific techniques for geospatial services and localization) that dynamically produces maps of spatially defined data from geographical information, returning a digital map, as a representation of such information, suitable to be displayed on a browser-web. calibration of the graphic components (types and thickness of the lines, sizing of the texts according to information hierarchies). The case study has allowed the graphic definition of a frame of reference for the support and the restitution of the resilience first seen at the building level, then brought to the level of agglomeration of buildings, finally weighted as a summation within an entire homogeneous urban space.

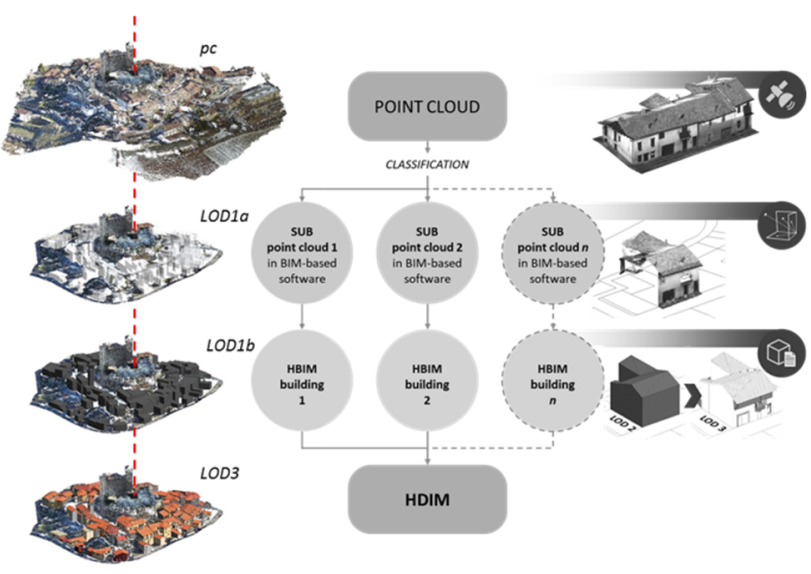

Figure 10. Schematic of the information system for planning and design management of cultural heritage

Despite the characteristics of economy and mathematical simplicity of a relational data model, to most professional engineers and architects, it appears at first glance as something foreign to the planning policies. Instead, it is a very useful conceptual reference framework for design elaboration: relational databases, even geometric ones, if connected to GIS/BIM systems, can become extremely efficient and productive tools: in a knowledge process, nothing is more important than the definition and discovery of the spatial and temporal relations that exist between the different parts of the work. It is necessary to think about the limits of the model and keep them in mind, i.e. to break it down and measure the degree of reliability of the individual parts.

\section{REFERENCES}

Adler, A.L. \& Urice, S.K., 2011. Resolving the disjunction between cultural property policy and law a call for reform. Rutgers Law Review, 64(1), pp. 117-163.

Aicardi, I., Chiabrando, F., Lingua, A.M., \& Noardo, F., 2018. Recent trends in cultural heritage 3D survey: The photogrammetric computer vision approach. JOURNAL OF CULTURAL HERITAGE. 32(2018). 257-266.

${ }^{3}$ Web-GIS are the geographic information systems published on the web; in this way, Geographic Infromatio System (GIS) applications, traditionally dedicated to stand-alone users or in LAN environments, can be implemented on web servers allowing interaction through the internet with the territorial representation and with the data associated with it. 
Bosché, F., Ahmed, M., Turkan, Y., Haas, C. T., \& Haas, R., 2015. The value of integrating Scan-to-BIM and Scan-vs-BIM techniques for construction monitoring using laser scanning and BIM: The case of cylindrical MEP components. Automation in Construction, 49, 201-213.

Chiabrando, F., Danna, C., Lingua, A.M., Nardo, F., \& Osello, A., 2017. 3D roof model generation and analysis supporting solar system positioning. GEOMATICA. 71:3(2017), 137-153.

Deng, Y., Cheng, J. C., \& Anumba, C. (2016). Mapping between BIM and 3D GIS in different levels of detail using schema mediation and instance comparison. Automation in Construction, $67,1-21$.

Donato, V.; Giannetti, S.; Bocconcino, M. M., 2017. H-BIM and web-database to deal with the loss of information due to catastrophic events - The digital reconstruction of San Salvatore's Church in Campi di Norcia (Italy), In: ShoCK! - Sharing Computational Knowledge! - Proceedings of the 35th eCAADe Conference, pp. 119-128.

Dore, C., \& Murphy, M., 2012. Integration of Historic Building Information Modelling (HBIM) and 3D GIS for recording and managing cultural heritage sites. Virtual Systems and Multimedia (VSMM), 2012, 369-376.

Guo, B., Huang, X., Zhang, F., \& Sohn, G., 2015. Classification of airborne laser scanning data using JointBoost. ISPRS Journal of Photogrammetry and Remote Sensing, 100, 71-83.

Hernandez, J., Marcotegui, B., 2009. Point cloud segmentation towards urban ground modeling. In: The 5th GRSS/ISPRS Joint Urban Remote Sensing Event (URBAN2009). Shangai, China, pp. 1-5.

Hron, V., \& Halounová, L., 2015. Automatic Generation of 3D building models from point clouds. Geoinformatics for Intelligent Transportation, 109-119.

Kang, T. W., Park, S. H. \& Hong, C. H., 2016. BIM/GIS-based Data Integration Framework for Facility Management. GEOProcessing 2016, 100-105.

Lo Turco, M.; Bocconcino, M. M., 2017. Esattezza, molteplicità e integrazione nell'Information Modeling\&Management Exactitude, multiplicity and integration in Information Modelling \& Management, In: TECHNE, pp. 267-277.

Ma, Z., \& Ren, Y., 2017. Integrated Application of BIM and GIS: An Overview. Procedia Engineering, 196, 1072-1079.

Malinverni, E. \& Tassetti, A. N., 2013. GIS-based smart cartography using 3D modeling. ISPRS-International Archives of the Photogrammetry. Remote Sensing and Spatial Information Sciences. 1(2). 47-52.

Osello, A.; Rinaudo, F., 2016. Cultural Heritage Management Tools: The Role of GIS and BIM, In: 3D Recording, Documentation and Management of Cultural Heritage, pp 105124.

Pang, G., Qiu, R., Huang, J., You, S., \& Neumann, U., 2015, May. Automatic 3d industrial point cloud modeling and recognition. 14th IAPR international conference on machine vision applications (MVA), 22-25.
Poullis, C., 2013. A framework for automatic modeling from point cloud data. IEEE transactions on pattern analysis and machine intelligence, 35(11), 2563-2575.

Previtali, M., \& Latre, M. Á., 2018. A brokered Virtual Hub approach for the generation of web applications based on historical maps. Applied Geomatics, 10(4), 453-472.

Ruffino, P.A., 2018. Communication and visualisation methodologies for UNESCO sites, In: Rappresentazione/Materiale/Immateriale Drawing As (In)Tangible Representation, pp. 1397-1402.

Ruffino, P.A., \& Del Giudice, M. (2018). From geospatial data to information modeling. Dn Building Information Modeling, Data \& Semantics. 35-44.

Santoro, M., Nativi, S., \& Mazzetti, P., 2016. Contributing to the GEO Model Web implementation: A brokering service for business processes. Environmental Modelling \& Software, 84, 18-34.

Spallone, R., Piano, A., \& Piano, S., 2016. BIM and cultural heritage: multi-scalar and multi-dimensional analysis and representation of an historical settlement. The case study of Montemagno, a New Village in Piedmont. DISEGNARE CON..., 2016, 1-12.

Sullivan, A.M., 2016. Cultural Heritage \& New Media: A Future for the Past. J. Marshall Rev. Intell. Prop. L., 15, 604, pp. 605645 .

Tobias, P., 2015. An Investigation into the Possibilities of BIM and GIS Cooperation and Utilization of GIS in the BIM Process. Geoinformatics FCE CTU, 14(1), 65-78.

Tommasi, C., Achille, C., \& Fassi, F., 2016. From point cloud to BIM: a modelling challange in the Cultural Heritage field. International Archives of the Photogrammetry, Remote Sensing and Spatial Information Sciences, 429-436.

Thomson, C., \& Boehm, J., 2015. Automatic geometry generation from point clouds for BIM. Remote Sensing, 7(9), 11753-11775.

Vacca, G., Quaquero, E., Pili, D., \& Brandolini, M., 2018a. GISHBIM Integration for the management of historical buildings. International Archives of the Photogrammetry, Remote Sensing \& Spatial Information Sciences, 42(2).

Vacca, G., Quaquero, E., Pili, D., \& Brandolini, M., 2018b. Integrating BIM and GIS data to support the management of large building stocks. International Archives of the Photogrammetry, Remote Sensing \& Spatial Information Sciences, 42(4).

Xu, C., Wang, Y., Tan, T., \& Quan, L., 2004. Automatic 3D face recognition combining global geometric features with local shape variation information. Sixth IEEE International Conference on Automatic Face and Gesture Recognition, 2004, 308-313.

Zhang, L., Li, G., Zhang, C., Yue, H., \& Liao, X., 2018. Approach and practice: integrating earth observation resources for data sharing in China GEOSS. International Journal of Digital Earth, 1-16.

Revised March 2019 